\title{
Design of Hybrid Mobile Communication Networks for Planetary Exploration
}

\author{
Richard L. Alena, John Ossenfort, Charles Lee, Edward Walker, Thom Stone \\ NASA Research Center \\ ARC MS 269-4 \\ Moffett Field, CA 94035 \\ 650-604-0262 \\ richard.l.alena@nasa.gov
}

\begin{abstract}
The Mobile Exploration System Project (MEX) at NASA Ames Research Center has been conducting studies into hybrid communication networks for future planetary missions. These networks consist of space-based communication assets connected to ground-based Internets and planetary surface-based mobile wireless networks. These hybrid mobile networks have been deployed in rugged field locations in the American desert and the Canadian arctic for support of science and simulation activities on at least six occasions. This work has been conducted over the past five years resulting in evolving architectural complexity, improved component characteristics and better analysis and test methods. A rich set of data and techniques have resulted from the development and field testing of the communication network during field expeditions such as the Haughton Mars Project and NASA Mobile Agents Project.
\end{abstract}

This paper will define design, analysis and test methods for hybrid mobile communication networks, identifying the key issues and constraints that affect performance in both the radio frequency (RF) and network engineering disciplines. Previous work by the MEX team has addressed the architecture and detailed analysis of wireless networks including the results of field tests. We will continue the analysis using a new $802.11 \mathrm{~b}$ backbone utilizing two repeaters that significantly increase range and coverage but greatly increase latency, which reduces overall network throughput. The addition of a satellite link can result in significant additional throughput loss due to light-speed delays in the space segment interacting with variable latencies in the multi-hop wireless network. The paper will analyze and present RF domain field test results combined with network performance metrics which describe a comprehensive approach for designing and optimizing future hybrid mobile networks. $\overline{10-7803-8155-6 / 04 / \$ 17.00 \text { (c) } 2004 \text { IEEE }}$

2IEEEAC paper \#
TABLE OF CONTENTS

1. INTRODUCTION

1

2. COMMUNication Archtecture OVERVHEW 2

3. Radio Frequency Domain Analysis 3

Theoretical Link Margin Calculations 3

WLan Product Calibration Curves 4

Fresnel Zone and Antenna Height 10

Orher RF Domain EfFects 10

4. Media Access Control Packet Domain 11

5. Internet Protocol Domain 12

Network Throughput Test Results 12

Latency and Window SIZE $\quad 16$

6. WLan Design and Deployment 16

7. Conclusions 19

REFERENCES

BIOgraphiEs

20

\section{INTRODUCTION}

The Intelligent Mobile Technologies (IMT) group at NASA Ames Research Center has been providing the basic computational and communication infrastructure required for the conduct of sophisticated planetary exploration simulations in remote rugged field locations around the globe. These simulations focus on both exploration technology development such as autonomy software and the operations concept development for missions involving mixed human and robotic teams. This work was initiated in 1998, and the IMT group has been involved in no less than eight different field expeditions to the Canadian high Arctic and the American Southwest deserts. Despite the differences in temperature, these locations are remarkably similar in their barren, harsh environments, supporting only occasional plant life in distinct ecological niches but providing a rich environment for the study of geology and biology relevant to extreme environments. In fact, NASA scientists select these "Mars analog" sites based on their similarity to anticipated Martian terrain and environmental conditions. An important selection criterion is the quality of science that can be performed at these earth sites - relevant to current earth science goals and the goals of astrobiology, the study of life on other planets. 
The Mobile Exploration System (MEX) is an IMT project that develops increasingly capable infrastructure for these field science surveys and exploration technology simulations. Both activities can occur in concert during expeditions such as the Haughton-Mars Project [1] and the Mobile Agents Project [2]. The MEX infrastructure models a communication and computational system that provides connectivity and computing functions between astronauts conducting a field science survey in a remote location to a habitat module acting as the primary planetary base and finally over a space link to earth-based mission control and science centers. MEX attempts to construct advanced capability using current technology to meet the goals of both high-fidelity simulations and the support needs for conduct of theses simulations by providing a satellite based wide-area network (WAN) uplink to a collection of computing assets at the habitat and extending this connectivity to remote field locations using wireless network (WLAN) technology.

The MEX 2003 field configuration is shown below (Figure 1) and illustrates the use of two repeaters to form a long-haul backbone to remote survey sites up to 5 miles away. The backbone terminates at the All-Terrain Vehicle (ATV) used to host the major computing and sensing assets. The ATV creates a local 802.11 b cluster which supports the astronauts, wearing computing and communication backpacks and various other computers and sensors. The Mobile Agents simulation scenario has the astronauts issuing voice commands to the astro backpack which are then executed by distributed software agents to take pictures, command rovers and transfer data.
Previous work in this area has resulted in papers that describe hybrid architectures for planetary communications and the test methods applicable to mobile networks. This paper is a continuation of this work, now extended to incorporate the habitat-wired networks and the space-based satellite links to the Internet. There is an interaction that occurs between such disparate communication systems that can significantly degrade performance if not properly addressed. Specifically, high latency on the space-based links can interact with the variable latency on the WLANs and result in low TCP/IP throughput. Solutions resulting from architectural improvements and tuning of the links are presented. Extensions of these methods to longer light-speed delays typical of satellite communication links are introduced. Finally, the results of field deployment and testing of the MEX $802.11 \mathrm{~b}$ wireless backbone is presented and compared to the results of other WLAN products tested earlier. In this paper, we attempt to illustrate how the general design of hybrid complex communication systems can only be addressed by concurrent solutions in the radio frequency domain, the WLAN packet domain and the TCP/IP domain.

\section{COMMUNICATION ARCHTTFCTURE OVFRVIEW}

The MEX communication architecture is intended to be a prototype for any future network system that could be designed and used for planetary exploration. It incorporates all the major elements: Earth-based Wide Area Networks, spacebased satellite links, habitat vehicle wired networks, habitat vehicle wireless networks, planetary-surface wireless backbones and cluster WLANs supporting field survey vehicles,

Figure 1 - Mobile Exploration System Field Configuration 2003

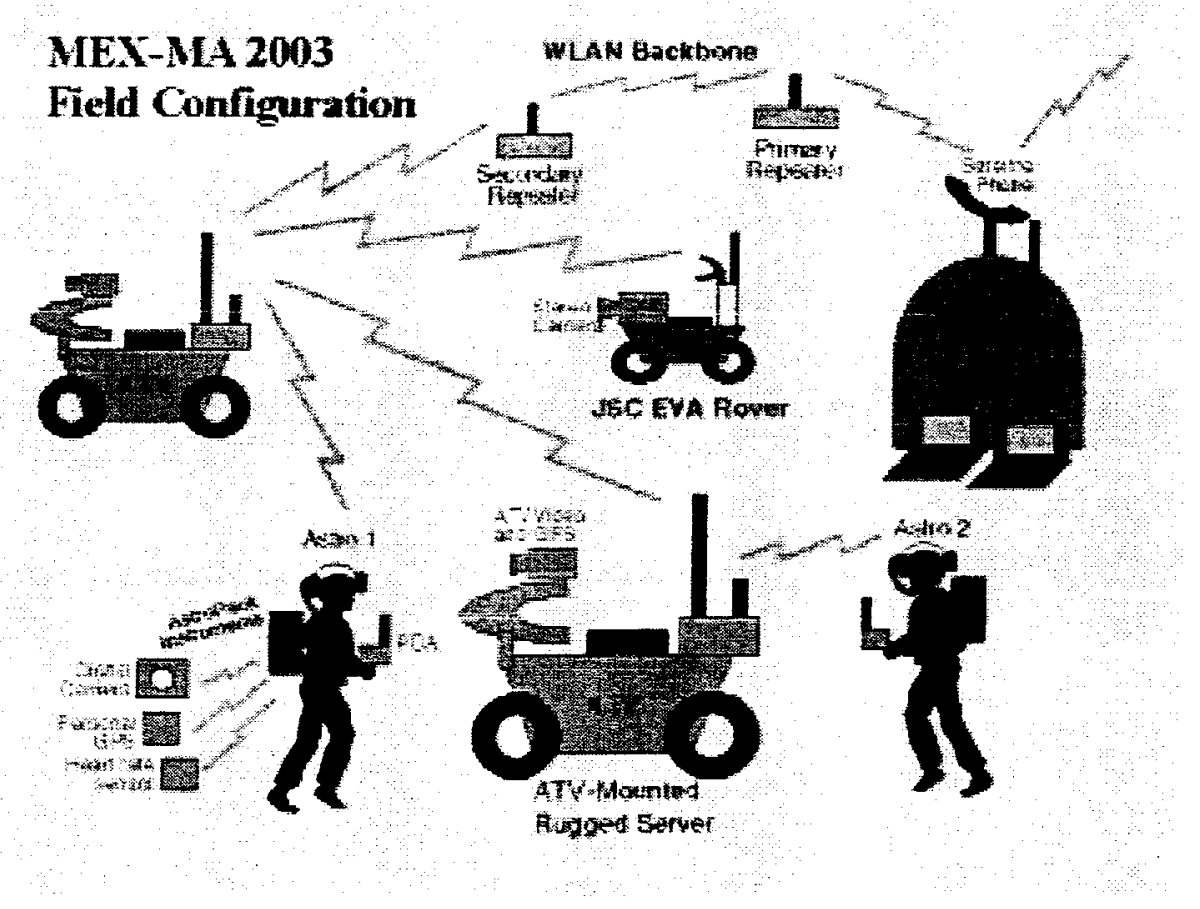


space suits and instruments. It provides all the services required for mission situational awareness and direction, scientific data acquisition and analysis and all system and personnel support services. In addition, it provides a superset of services for support of the field expedition itself and for the conduct of the simulations such as internet telephony, video teleconferencing and Internet access and e-mail. For example, as three people are involved in conducting a real-time simulation of science surveys in the field, up to 20 people are involved in supporting the system and software functions needed to conduct the simulation.

Applications required in the field such as Voice over Intemet Protocol (VoIP), and Internet-based video conferencing and streaming video can drive network bandwidth and latency requirements. These applications require near real time response and have the following attributes: Packet voice and video use the User Datagram Protocol (UDP) meaning that this data is not subject to the delays inherent in Transmission Control Protocol (TCP) which must receive a reply after a series of packets are sent. UDP sends data in a continuous stream without regard to the state of the receiver. A small data loss (or errors) can also be tolerated with voice and video transmissions, resulting in some inconsequential loss of quality. Jitter (variable delay between packets), caused by network latency and out of sequence packets, can cause problems with voice and video processing that depends on synchronous packet transfer.

Voice and streaming video are basically half duplex, meaning that they are transmitted in one direction at a time. With VolP there can be more than one conversation at once meaning that packets could be flowing in both directions (full duplex). Video conferencing is always full duplex since images are flowing in both directions so the party at either end can see the other. This factor is especially important for transmission over WLANs, which are half duplex in nature with highly variable latency. Another problem is that TCP and UDP are not very good at sharing network links. TCP tends to cut back on its transmission rate when packets are dropped and routers tend to drop UDP packets in undesirable ways when congestion occurs. VoIP products have developed means to compensate for echo and the long but consistent delay of satellite links. Video conferencing equipment however can be problematic because satellite links are usually asymmetrical having different bandwidth in each direction. NASA ARC is now using network monitoring to determine the requirements and problems associated with these applications in the field environment. There exist Quality of Service techniques that can mitigate the problems with sending voice and video on constrained WLAN links, to be explored in future papers.

An end-to-end analysis of the communication paths required to acquire a digital picture from a camera in the astronauts hand to a scientist located on earth shows how many links and computational nodes are involved in this data transfer. The computing nodes are critical to communication system function and performance because they can buffer and store data between link segments, with a dramatic effect on communication system performance. The data path is essentially from camera through camera interface (wireless Bluetooth or USB) to astronaut computer to ATV cluster WLAN, to ATV computer, to MEX backbone WLAN, to habitat network and computers to satellite dish transceiver (via wireless link) to satellite to ground station to Intemet router through Internet to Science Center network to scientist workstation. This is a total of at least ten discrete links or hops! The MEX network architecture is designed to be a unified subnet, with the ATV and astronaut nodes accessible directly from the habitat. Actual systems for Mars exploration will be even more complicated, but the design issues are similar - allowing us to define and test prototypes, which have relevance to systems to be designed ten years hence.

This paper analyzes the wireless network backbone in detail, using RF domain analysis to determine the range and coverage of a given link segment. It looks at theoretical and measured RF received signal levels using various combinations of antennas and compares these reference values to field-test values during deployment in the Utah desert. Other factors affecting link performance in the RF domain, such as antenna height and multipath, are discussed in detail. Then the effects of Media Access Control ( 802.11 b) layer on digital packet transport are analyzed, looking at the effects of bit error rate on throughput. Finally, the paper will show several measurement methods used at the Internet Protocol transport layer to determine network throughput under various conditions using single and double repeater systems. The effects of latency on throughput are detailed, along with mitigation techniques such as window size adjustment and buffering. Detailed quantitative analysis of RF signal strength correlated with network throughput and latency is presented and compared to the field-test results.

\section{Radio FReQuency DomaIn ANaLysis}

Theoretical Link Margin Calculations:

Wireless networks use RF signals (or optical signals) to communicate information from point to point. The theory of RF propagation is well developed and understood and can be applied directly to WLANs. Basically, a transmitter with a certain amount of output power sends its signal through an antenna that focuses the radio signal into a directional beam, amplifying it in relation to an isotropic radiator. This effective radiated power (EIRP) is the radio signal power leaving the transmitter antenna. When propagating from transmitter to receiver it suffers significant signal loss from attenuation caused by spread of the radio beam with distance (d), a phenomenon known as free-space-path-loss (Ls), given by the following equation in $\mathrm{dB}$ :

Ls $=36.57+20\left(\log \left(d / 5280^{*} 39 / 12^{*} 2442\right)\right.$

where $d$ is in meters 
The receiving antenna collects the remaining signal at the end of the propagation path and passes it to the receiver. The receiver has a certain sensitivity that allows it to interpret the information coded into the RF signal. The received radio signal strength must be greater than the specified sensitivity for the information to be recovered. The sum total of these factors governing radio transmission is termed the "link budget" and is given by the Friis equation:

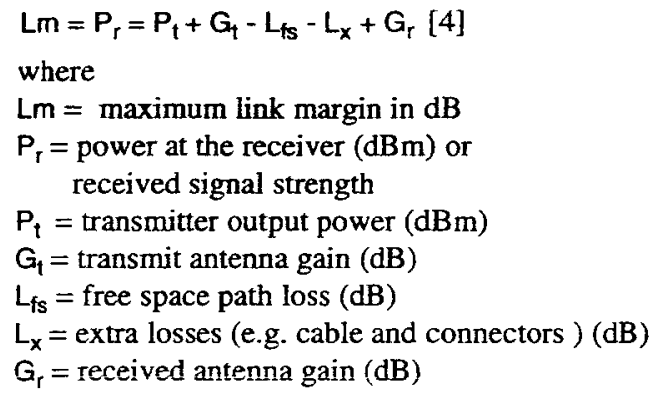

The result of this calculation is a value of received signal strength at the far end of the link segment. Since antenna gain and cable losses are constant, a plot of $\operatorname{Pr}$ versus distance will produce an exponential fall-off of received signal strength with distance, producing the attenuation curve based on RF propagation theory. The units used for these calculations are power decibels $(\mathrm{dBm})$ described by the equation:

$$
\begin{aligned}
& \mathrm{Pt}=10 \log \mathrm{Po} / \mathrm{Pr} \\
& \text { where } \\
& \mathrm{Po} \text { is power output } \\
& \mathrm{Pr} \text { is reference power of } 1 \mathrm{~mW} \text {. }
\end{aligned}
$$

This means a $100 \mathrm{~mW}$ transmitter would be characterized as having a $+20 \mathrm{dBm}$ output signal. A decrease of only $3 \mathrm{dBm}$ in signal strength implies that half the original power is now available, an indication of the logarithmic nature of these numbers. Antenna gain is expressed as the decibel equivalent compared to an isotropic (equal radiation in all directions) radiator in units of $\mathrm{dBi}$. Cable and other parasitic losses are similarly expressed as $\mathrm{dB}$ losses per meter or loss per connector. These parasitic losses can be a significant factor, especially at frequencies of $2.4 \mathrm{GHz}$, used by $802.11 \mathrm{~b}$ WLANs.

The maximum distance that can be supported by a communication link is proportional to the square root of the power received - if we increase the received power by $6 \mathrm{~dB}$, or quadruple it, we will double the maximum distance. This is expressed in the formula:

$$
\begin{aligned}
& \left.\mathrm{Dm}=300 / 2442 \cdot \exp \left((\mathrm{Lm}-22-\mathrm{Rm}) / 6^{*} \ln 2\right)\right) \\
& \text { where } \\
& \mathrm{Dm} \text { is distance in meters } \\
& \mathrm{Lm} \text { is maximum link margin in } \mathrm{dB} \\
& \mathrm{Rm} \text { is desired receiver signal to noise margin, } \\
& \text { typically } 10 \mathrm{~dB} \text { over sensitivity rating }
\end{aligned}
$$

For example, we will model our MEX field repeater link, which used an $18 \mathrm{dBi}$ parabolic dish at both ends, with $100 \mathrm{~mW}$ of transmit power and about $3 \mathrm{~dB}$ of cable and connector loss at each end. The calculation yields a maximum distance of 11.13 miles or $18.07 \mathrm{Km}$, as shown in Table $\mathbf{1}$, below.

These equations can be used to predict received signal strength and therefore performance at specific distances for various radio and antenna combinations. They can also predict maximum distance for a given link configuration and form the basis of raudio system design. Howcver, many other factors can affect radio propagation and we apply pragmatic methods as well as theory.

\section{WLAN Product Calibration Curves}

To determine how well the radio engineer has setup a given link segment, one can measure the performance of a WLAN system and compare these test results to the theoretical curve of received signal strength versus distance. There should be a reasonable correlation with theory and if not, there is some problem with the setup. However, the measurement tools generally provided with the WLAN systems are not calibrated and much too inaccurate for this purpose. Each WLAN product line comes with software tools to assist the radio engineer in setting up and analyzing the RF domain characteristics of the WLAN under actual conditions by measuring RF signal strength and signal quality under controlled conditions. Most products provide qualitative values and these measurements are generally inaccurate. Some products provide quantitative values, but these values seldom correlate with the values used in the theoretical calculations described above. The reason for this discrepancy is different assumptions regarding antennas, additional losses, non-linearity of the RF circuits and other factors not included in the WLAN tool's calibration.

Table 1. Grid Dish-to-Grid Dish Antenna Link Calculation

\begin{tabular}{c|c|c|c|c|c|c|c}
\multicolumn{2}{c|}{ Antenna 1 } & Cable 1 & \multicolumn{2}{c|}{ Antenna 2 } & \multicolumn{2}{c|}{ Cable 2 } & \multicolumn{2}{c}{ Max. Distance w/ 10dB margin } \\
\hline Model & Gain dBi & Loss dB & Model & Gain dBi & Loss dB & \multicolumn{1}{c}{ Miles } & Km \\
\hline Grid Dish & 18 & 3 & Grid Dish & 18 & 3 & 11.13 & 18.07 \\
\hline
\end{tabular}




\section{MEX Link Testing}

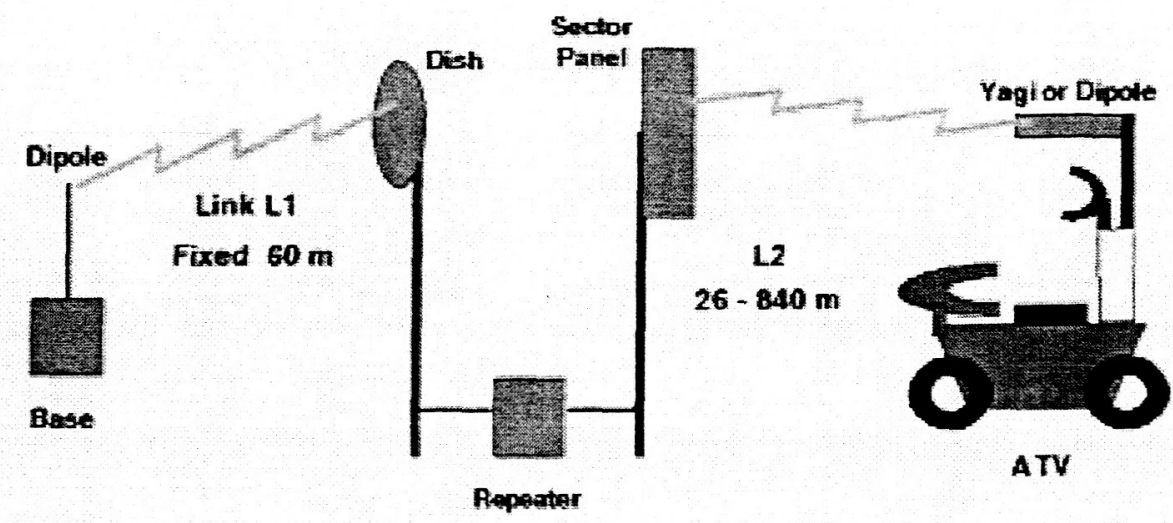

Figure 2 - MEX Link Test Range Diagram

The RF domain signal strength value measured using these tools needs to be correlated with the numbers obtained from the theoretical RF link equations, which are correct from the radio physics viewpoint. Then the numbers obtained from the WLAN tools can be translated into the correct physical units and accurately used to predict link performance in various field conditions and for determining how well a given link has been setup and is performing.

We use measurements taken in the field under controlled conditions to create calibration curves for the RF site survey tools provided with each WLAN product line. The controlled conditions are precise antenna and cable construction and alignment, a test range free of interference or physical obstructions and a careful procedure for conducting the tests. We then use certain techniques to normalize these WLAN product values to the theoretical calculations and values. This yields the ability to apply the crude WLAN tools quantitatively for estimating maximum range or for determining how well a given installation is performing. The process is to curvefit the WLAN tool received signal graph to the theoretical received signal graph, taking into account certain factors, such as parasitic losses and atmospheric absorption of the RF signals. The parasitic losses are constants and appear as an offset between the two curves. The additional loss due to atmospheric absorption is proportional to distance and appears as a change in slope between the two curves. Determining the values of these additional 2 nd order effects can be difficult if the two graphs are significantly discrepant.

Our radio test range is shown schematically in Figure 2 with Link 2 (L2) being the one under test. Link 1 (L1) consisted of a fixed link between the root bridge indoors inside our lab and the first repeater approximately 60 meters away, located across the street outside of the building but well within line- of-sight of the root. The root bridge, using a single rubber $2.2 \mathrm{dBi}$ dipole antenna, communicated to the first repeater equipped with a directional $18 \mathrm{dBi}$ small dish grid antenna to complete $\mathrm{L} 1$. The Cisco 350 Bridges were equipped with two RP-TNC connectors for antenna attachment, and a low-loss cable of about 15 feet length was used to connect the repeater box to the antennas, which were mounted on separate antenna masts at the first repeater site just outside our building. The two masts were about six feet apart and the antennas were mounted about 6 feet high.

I ink 2 consisted of a $13 \mathrm{dBi}$ sector panel antenna at the first repeater site aimed down the length of a long street perpendicular to Link 1. The elevation angle was zero degrees, assuring optimal transmission to the ATV antenna mounted at a similar height. Buildings on either side but offset from the street allowed for an open thoroughfare of 60-90 meters down most of the street, finally opening up entirely on the far end of Link 2. The distance of Link 2 varied with each test point that were located at approximately 30 meters, 80 meters, 160 meters, 320 meters and 500 meters from the first repeater. The farthest measured point from the first repeater was 840 meters away and located on the opposite side of a iarge field, with mostly low-lying brush between this point and all other points located along the street. The only other objects to pose a possible obstacle were parked cars and the occasional stop signs along the street. Our test platform was the ATV, with two main antenna types, dipole and Yagi mounted on an adjustable arm above the rear platform around six feet high. 


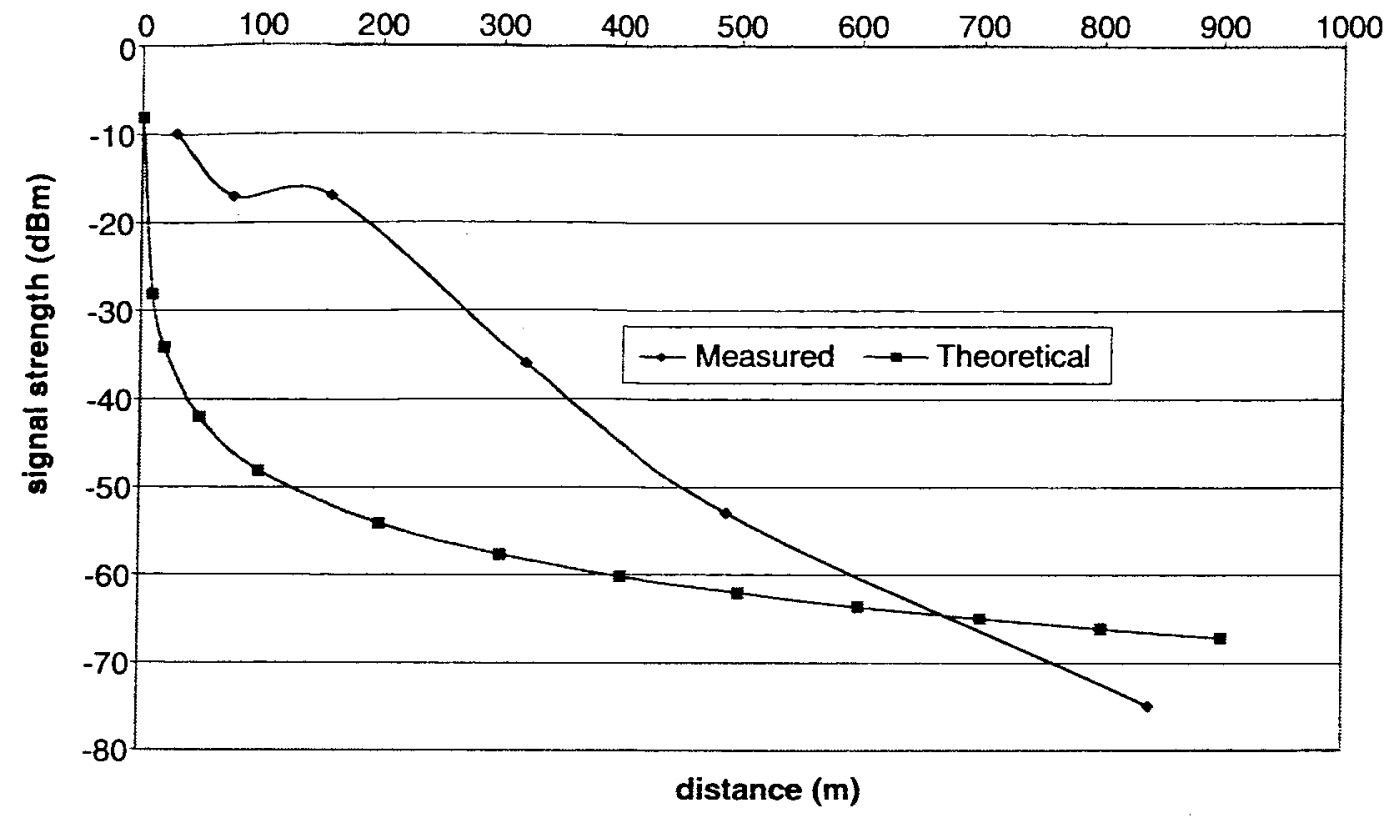

Graph 1 - Signal Strength Calibration - Sector Panel to Dipole

We will use three examples to illustrate this technique for the Cisco 350 Bridge units used for the MEX backbone. Graph 1 , above, shows the calibration curve obtained in the field using the antenna-pointing tool on the Cisco 350 Bridge, which produces RF signal strength values in $\mathrm{dBm}$ units, not necessarily accurate from a physics perspective. It also produces a signal quality number expressed as a percentage, with $100 \%$ being excellent quality. Quality is generally the ability to interpret the data modulated into the radio signal and is affected by multipath interference and other signal scrambling effects. Signal strength is affected primarily by path loss for a given antenna combination with fixed parasitic losses. The values for RF signal strength obtained from theory are shown on the same graph. Note that the theoretical received signal strength conforms to the expected exponential dropoff with distance.
Graph 1 illustrates the difference between the measured received signal strength value using the Cisco antenna-pointing tool and the theoretical link calculation. Visual comparison shows that the scale and offset of the two curves are dramatically different, with the measurements showing much higher values then theory. In this case, the two curves are so different that a lookup table approach should be used to interpret the numbers. If we want the theoretical equivalent we lookup the measured value on its curve and simply read the corresponding theoretical value by going down vertically on the graph.

The first case tests link L2 between the repeater and ATV using a sector panel antenna with $13 \mathrm{dBi}$ gain on the repeater and an omni directional dipole antenna with $5 \mathrm{dBi}$ gain on the ATV. The numbers for the link calculation are shown in Table 2, yielding a modest 1.4 miles of distance for this configuration.

Table 2. Sector Panel to Dipole Antenna Link Calculation

\begin{tabular}{|c|c|c|c|c|c|c|c|}
\hline \multicolumn{2}{|c|}{ Antenna 1} & Cable 1 & \multicolumn{2}{|c|}{ Antenna 2} & \multirow{2}{*}{$\begin{array}{l}\text { Cable } 2 \\
\text { Loss dB }\end{array}$} & \multicolumn{2}{|c|}{ Max. Distance w/ 10dB margin } \\
\hline Model & -Gain dBi & Loss $d B$ & Model & Gain $\mathrm{dBi}$ & & Miles & $\mathrm{Km}$ \\
\hline Sector & 13 & 3 & Omni & 5 & 3 & 1.39 & 2.26 \\
\hline
\end{tabular}




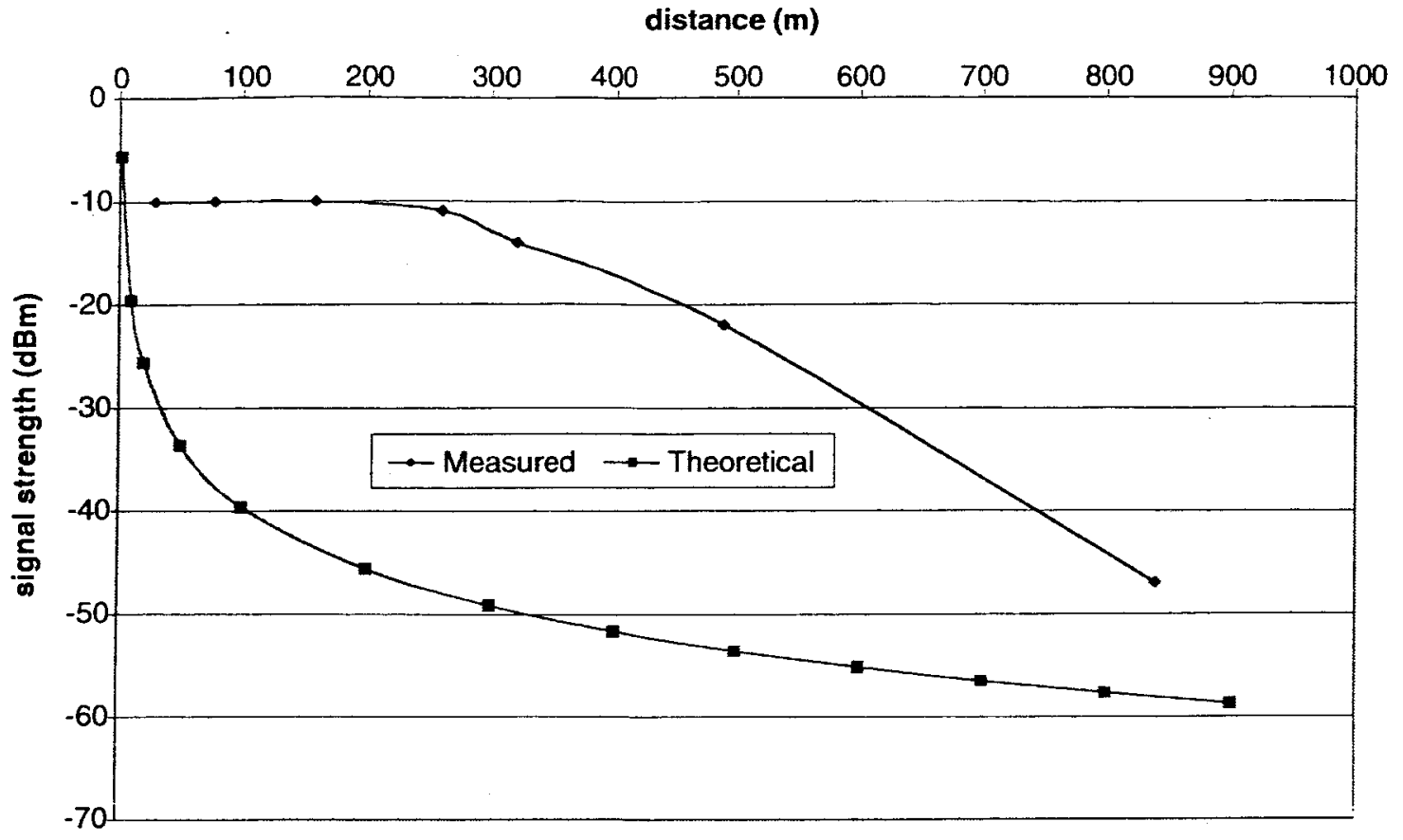

Graph 2 - Signal Strength Calibration - Sector Panel to Yagi

For the second case, the dipole is replaced with a Yagi directional antenna, yielding an increase in link gain and therefore maximum range. This is shown in the link calculation table (Table 3, below). A difference in $8.5 \mathrm{~dB}$ yields more than double the maximum distance, as expected, since a $6 \mathrm{~dB}$ increase in signal strength will double the range according to the link equation.

Graph 2, above, is for Case 2, using a sector panel antenna with $13 \mathrm{dBi}$ gain for the repeater and a $13.5 \mathrm{dBi}$ Yagi antenna for the ATV. When we compare this graph to the previous one, the first thing to observe is that the two theoretical curves are identical, just offset by $8 \mathrm{~dB}$, reflecting the higher gain of the Yagi antenna. Fixed differences in link gain are revealed as offsets between the two received signal curves. However, when the two measured curves are compared, there is no fixed offset. At 320 meters, the offset is $22 \mathrm{~dB}$, at $480 \mathrm{~m}$ it is $31 \mathrm{~dB}$ and at $840 \mathrm{~m}$ it is $28 \mathrm{~dB}$. The measured values are much higher than the theoretical values generally and fail to exhibit the exponential drop off expected, especially at short distances.
In fact, the curve for Case 2 is flat until 280 meters! After that, the curve tends to fall off linearly with distance, again the opposite of theory, which has it flattening out at larger distances. Obviously something is very wrong with the numbers coming from the Cisco WLAN tool. Not only are the numbers wrong, the curve has the wrong shape.

How can we validate our table lookup scheme, which provides normalized theoretical values from the measured values provided by the WLAN tool? We are comparing two links where the only difference is one antenna, allowing direct comparison of the two sets of numbers from the two different link configurations. This provides a crosscheck on the accuracy of the technique. The reduction of overall link gain caused by using a lower-gain omni-directional dipole antenna at the ATV is evident from inspecting the two graphs which shows higher measured and theoretical numbers with the Yagi.

Table 3. Sector Panel to Yagi Antenna Link Calculation

\begin{tabular}{c|c|c|c|c|c|c|c} 
Antenna 1 & & Cable 1 & Antenna 2 & & Cable 2 & \multicolumn{2}{c}{ Max. Distance w/ 10dB margin } \\
\hline Model & Gain dBi & Loss dB & Model & Gain dBi & Loss dB & Miles & Km \\
\hline Sector & 13 & 3 & Yagi & 13.5 & 3 & 3.71 & 6.03 \\
\hline
\end{tabular}




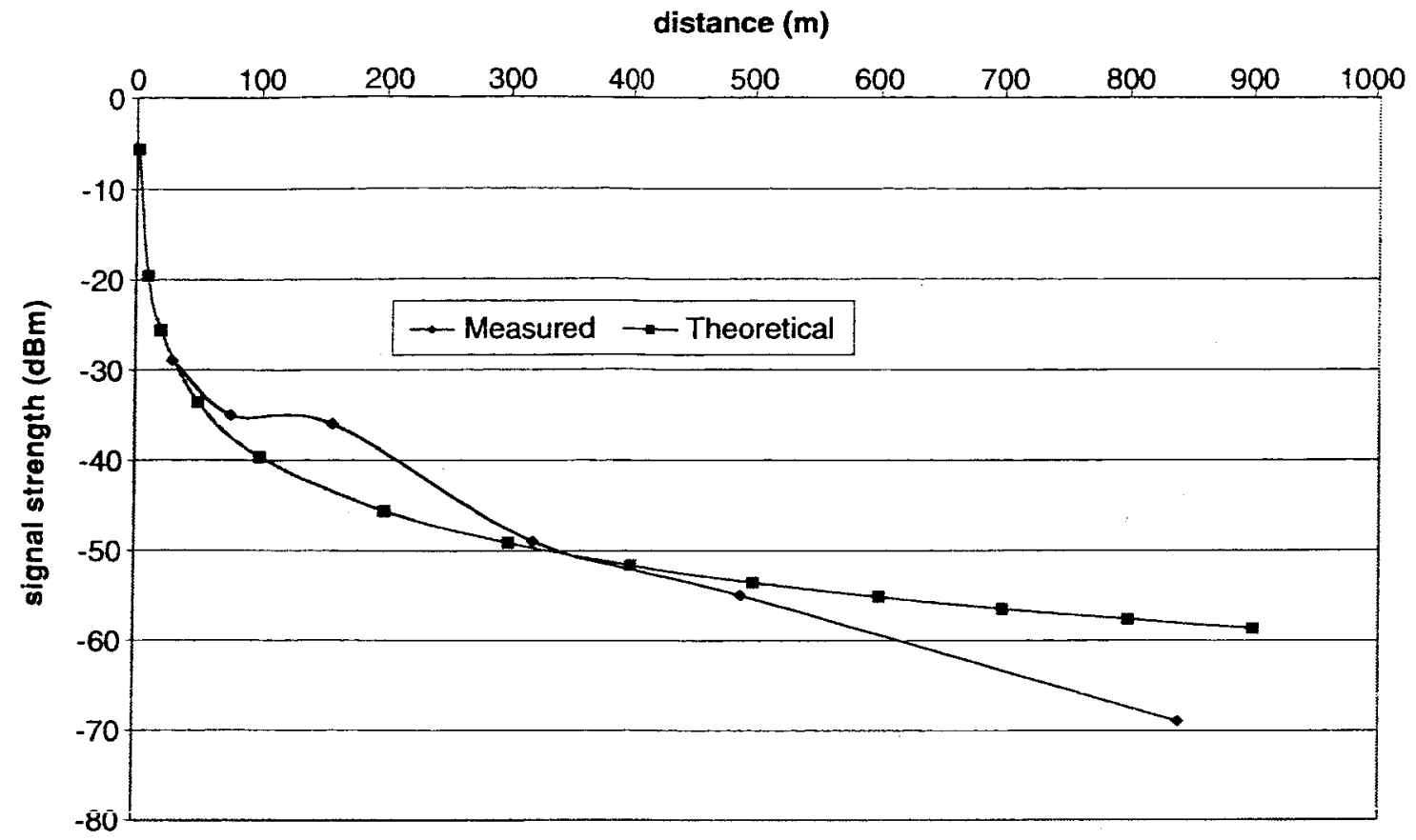

Graph 3 - Signal Strength Calibration - Sector Panel to Yagi, New Firmware 12.03

To demonstrate the accuracy of the normalization technique using table lookup, we chose two data points in the linear range of each graph, representing $-22 \mathrm{dBm}$ and $-47 \mathrm{dBm}$ measured from the Cisco bridge. Table 4 , below, shows the correlations with distance and theoretical received signal strength.

The correlation of theoretical received signal strength to Cisco tool received signal strength is good within $2 \mathrm{dBm}$ for very different link configurations. Note that the reduction in distance between the two configurations is nearly what is expected from a reduction of $8.5 \mathrm{~dB}$ of link gain. This validates both data sets and the calibration technique.

An additional crosscheck is provided by means of a firmware upgrade to the Cisco bridges, which could affect the RF signal levels, since these are provided through the firmware. We needed to obtain calibration curves of the firmware used in previous field tests, so that the field data could be analyzed, but for future field tests, we need to use the upgraded firmware. Graph 3, above, shows a repeat of the Sector Panel to Yagi case 2 test, but using firmware version 12.03 rather than version 11.03. The measured curve on the graph is completely different, even though the theoretical curve is the same as Case 2. The firmware upgrade did address the calibration of the RF signal strength measured value and actually achieved a reasonable fit with the theoretical curve! One significant difference is that the measured value is now lower than the theoretical value, below $-50 \mathrm{dBm}$, which still necessitates using this normalization technique even with the firmware upgrade. We determined that the bottom value of RF Signal Strength is $-91 \mathrm{dBm}$, corresponding to the sensitivity specification for the Cisco Bridge at its lowest data rate.

The gold standard for RF signal measurements is the spectrum analyzer, which produces a plot of RF energy versus frequency over the band of interest. The MEX project is acquiring such an instrument (which is quite expensive for frequencies above $1 \mathrm{GHz}$ ) and will compare these results to this instrument at a later date. These instruments use calibrated antennas to sample the radio signals they are measuring, and this is a source of significant error due to misalignment or other errors in measurement technique. Therefore, the methods outlined in this section are also relevant to validate the measurements obtained using precision instruments. The most important contribution of this analysis method is the ability to correlate multiple instruments. The technique for doing this is to use a calibrated test range and procedure.

Table 4. Comparison of Normalization Technique for Case 1 and Case 2

\begin{tabular}{c|c|c|c|c}
\multirow{2}{*}{ Cisco Recv Sig $(\mathrm{dBm})$} & \multicolumn{2}{|c|}{ Sector panel to Yagi } & \multicolumn{2}{c}{ Sector panel to Dipole } \\
\cline { 2 - 5 } & Distance $(\mathrm{m})$ & Theo Recv Sia & Distance $(\mathrm{m})$ & Theo Recv Sia \\
\hline-22 & 490 & -53.4 & 200 & -54 \\
\hline-47 & 840 & -58 & 400 & -60 \\
\hline
\end{tabular}




\begin{tabular}{|c|c|c|c|}
\hline $\begin{array}{c}\text { Wireless Link } \\
\text { Distance in Miles }\end{array}$ & $\begin{array}{c}\text { Approximate Value " } F \text { " } \\
\text { in Feet }(60 \% \text { Fresnal Zone } \\
\text { at } 2.4 \mathrm{GHz})\end{array}$ & $\begin{array}{l}\text { Appoximate Value "C" } \\
\text { (Earth Curvature) in Feet }\end{array}$ & $\begin{array}{c}\text { Value "H" } \\
\text { Antenna Mounting Height } \\
\text { with No Obstructions in Feet }\end{array}$ \\
\hline 1 & 10 & 3 & 13 \\
\hline 5 & 30 & 5 & 35 \\
\hline 10 & 44 & 13 & 57 \\
\hline 15 & 55 & 28 & 83 \\
\hline 20 & 65 & 50 & 115 \\
\hline 25 & 72 & 78 & 150 \\
\hline \multicolumn{4}{|c|}{ Total Distance } \\
\hline $\begin{array}{c}\text { Antenna } \\
\text { Height }\end{array}$ & & Fresnel $@ 60 \%$ & \\
\hline
\end{tabular}

Figure 3 - Fresnel Zone and Antenna Height

\section{Fresnel Zone and Antenna Height}

Another primary factor for RF propagation is the Fresnel zone, which has implications for antenna height and line-of-sight considerations. The Fresnel zone is the area required to efficiently propagate the RF energy so an antenna can receive it. The propagating wavefront actually has to occupy a certain area for enough energy to be transmitted, and this area is expressed as a circle with a given radius perpendicular to the propagation direction. This radius describes the Fresnel zone area and is proportional to the wavelength of the RF energy and proportional to the distance from the transmitter. Figure 3, above, has a table for Fresnel zone size at various distances for the $2.4 \mathrm{GHz}$ WLAN band. The picture illustrates how Fresnel zone affects the required height of the antenna at various distances. Basically, the antenna has to clear the earth bulge between the transmitter and receiver by at least the Fresnel zone defined in this case as being the size of the area required for $60 \%$ of the transmitted energy to get to the receiver. Partial blockage of the Fresnel zone will result in lower energy in the link, with concurrent reductions in RF signal values and link performance. Our test range barely conforms to this requirement at the longest test distance of 840 meters. Note how tall the antennas need to be to cover substantial distance even at this high frequency.

\section{Other RF Domain Effects}

There are other physical effects on radio propagation relevant to WLAN design, which are not accounted for in the theoretical link calculations. One such effect is atmospheric loss. which scales with distance through the atmosphere. This effect is particularly important in the $2.4 \mathrm{GHz}$ band, since this band is in the water window, where water vapor can absorb the incident RF energy. A previous paper attempted to define this loss and arrived at a value of $0.01 \mathrm{~dB} / \mathrm{m}$. [6] For the maxi- mum test distance, this would be about $8.4 \mathrm{~dB}$ of additional loss, a significant difference. Note that the measured curves show a larger drop off of received signal strength at larger distances and that this effect could contribute to this difference. These additional effects all reduce the link gain and therefore the maximum distance. The theoretical calculations really should be considered the best-case values. Future work with precision spectrum and power analyzers will attempt to define the atmospheric loss factor more precisely, since it is relevant for long distance links.

Another major effect is multipath interference, which has the effect of reducing the operating range of a WLAN by affecting the information content of the signal rather than its RF signal strength. When a link has high RF signal strength, but low bandwidth, this is a multipath effect in which a reflected wave interferes with a direct wave from the same transmitter, effectively overlaying part of the information content. Under extreme circumstances, this direct and reflected energy can setup standing waves where the RF signal strength varies dramatically by location, reaching peaks and nulls related to the wavelength of the RF energy. This effect is predominant in 802.11 bystems, since they offer no information redundancy which might mitigate this effect. In the field, when we observe a strong reflection situation, we also see a significant concurrent reduction in link throughput and reliability. The use of directional antennas can mitigate multipath distortion by selecting only the primary direct beam of energy from the transmitter.

Two other phenomena directly affect link gain. Antenna pointing accuracy is the angular alignment of one antenna to the other producing maximum gain. Accurate alignment will produce the specified gain of the antenna and misalignment will decrease it, perhaps drastically. The antenna radiation patterns show how accurate the pointing needs to be for both 
azimuth and elevation angles. Generally, mounts accurate to a fraction of a degree are desired for long-haul links, a real problem for mobile repeaters. The second phenomenon is the polarization direction of the electromagnetic wave. Certain antennas, such as sector panels, produce electromagnetic radiation that vibrates in only one direction, for example, vertically. The corresponding receiving antenna must also be capable of receiving the energy in this polarization plane. If it is horizontally polarized - for example, it will not "see" the vertically polarized signal. This can be responsible for low received signal strength.

\section{Media Access Control PaCket Domain}

The Media Access Control (MAC) layer specification for the Cisco WLAN is the IEEE 802.11b standard, corresponding to the 802.2 standard for wired Ethernet packets. The MAC layer is the physical transport layer for digital data. The MAC layer specifies the manner in which the physical medium for information transfer is used to provide multiple node access and for the transmission and interpretation of digital packet data. The 802.11b MAC specifies Carrier Sense Multi-access; clicnt device association and authentication to an access point; request-to-send and clear-to-send handshakes; and the format of the radio data packets and acknowledgements. This is similar to the Ethernet access protocol and packet format used for wired LANs but in the radio domain. Certain effects occur in WLANs that do not generally occur in wired LANs.

The first specification applied to physical data communication links is the Bit Error Rate, or BERR, which specifies the rate at which bits are corrupted by noise, interference or inability to correctly decode the information contained in the radio signal. For wired media, this can be on the order of only one lost bit per $10^{12}$ bits or a BERR of $10^{-12}$. However, for radio links this value is much higher, typically a loss of one bit per $10^{9}$ bits for a BERR of $10^{-9}$. Once a single bit is lost, the entire packet of data containing that bit needs to be retransmitted. Each packet contains about 1 Kbytes of data, or $8 \mathrm{Kbits}$ of data, therefore a single bit error will incur a cost of transmitting the corrupted $1 \mathrm{~KB}$ packet again. The time cost on an $11 \mathrm{Mbps}$ link would be about 800 usec. The net result of a high BERR is that many radio packets will need to be retransmitted, resulting in much lower network throughput and higher and more variable data transmission latency.

Multiple effects can cause high BERR, necessitating effective analysis of root cause to correct the problem. If the RF signal strength is too low, the receiver is unable to resolve the modulated data in the signal, and many bit errors can occur. This is what happens when the distance between transmitter and receiver is too large, or when antennae are misaligned or there is a significant obstruction of the Fresnel zone. However, low RF signal strength is not the usual cause of these types of problems. Multipath interference or distortion is usually responsible for high BERR in $802.11 \mathrm{~b}$ sys- tems. The 802.11 b system uses a direct-sequence spread spectrum modulation scheme for encoding the data into the radio signal. Essentially up to one third of the $2.40 \mathrm{GHz}$ to 2.483 $\mathrm{GHz}$ frequency band is used to transmit the full bit rate simultaneously. Interference in any portion of this active band will corrupt the bits corresponding to that portion of the RF spectrum, resulting in significant doss of data and high BERR. Multipath occurs when a reflected part of the transmitted radio signal interferes with a direct beam of the same transmitted signal. This will comupt certain segments of the radio signals in the spread spectrum resulting in loss of the corresponding information content. Although the effect occurs in the RF domain, its effects are apparent at the packet layer. This effect can be very severe for $802.11 \mathrm{~b}$, essentially shutting down the link despite very strong received signal levels.

A strong reflection from a building or hill can produce a very strong multipath effect with a corresponding large reduction in link throughput. A series of small reflections can also cause the same effect, but in a more subtle manner. The term for this is ground scatter, which occurs when part of the transmitted radio signal hits rocks, plants and other objects on the ground between the transmitter and receiver. These small objects scatter the radio signal by reflecting just a tiny portion of it. The aggregate sum of this scatter can reduce network throughput by increasing BERR. We have been trying to quantify the effects of multipath and scatter on throughput, but are having difficulty producing repeatable test conditions.

The instruments used for radio packet analysis are called protocol analyzers. They actually intercept and interpret each $802.11 \mathrm{~b}$ radio packet and can determine collision rate and BERR, and can measure latency of packet transmission. They can also identify access points and associated clients by MAC address. The MEX project has applied them in the field but has not performed the controlled testing needed to validate and interpret the results. This is a future effort.

The MAC layer is the physical foundation for data communications in networks. However, for WLANs, the MAC layer often obscures the actual performance of the WLAN because it will automatically retry transmission at the MAC packet layer in case of packet loss. Successful retransmission will only incur a delay in data transfer and will prevent the packet loss from being seen at the higher levels of the network stack. The MAC layer is responsible for all media access handshakes and for RF packet transfer and error handling, as well as repeater turnaround from transmit to receive mode. The timing of these processes varies from product to product and MAC layer software versions. The aggregate effect of these MAC layer timing interactions is difficult to predict, but have the effect of increasing latency, increasing latency variability and decreasing throughput if not optimal. However, there are ways to measure these effects indirectly by applying benchmark metrics at the transport layer. 
In fact, the method of predicting network throughput in complex WLAN systems is to use simulations of MAC layer function in tools such as OpNET. [7] Since the MEX project is primarily involved in providing functional WLAN capability in the field, our methods are the experimental equivalent of simulations, running the desired WLAN configuration under ideal conditions and extrapolating the results to the more complex field conditions encountered in planetary exploration simulations. This pragmatic approach produces results directly applicable to the WLAN product line being used and accounts for all variables, including interactions between network protocol stacks and applications. (As a side note, we have found significant similarities between different vendor's products based on the $802.11 \mathrm{~b}$ protocol, so we consider the test results generally applicable to WLAN design.)

\section{INTERnet PRotocol DomaIN}

The Transport Control Protocol for the Internet Protocol (TCP/ IP) implements data sockets for transfer of data between computers. It resides one level above the MAC physical transport layer and creates a mechanism for creating virtual logical connections between machines using an IP address:socket number tuple. This is the level at which application programmers deal with the network and is therefore accessible to applications that can be used for measuring network throughput and latency at the IP level. There are many tools to do this and we use a variety of techniques to crosscheck results. As in all complex systems, the exact method of measurement can affect the results.

One easy way to measure sustainable throughput is to download a file from an FTP server using an FTP client across the network link under test. Simply dividing the file size by the time gives us sustainable average throughput. The computers must be fast enough to serve the FTP data and consume the FTP data so that network performance, and not computer performance is the dominant bottleneck. We use file sizes of several Mbytes, which take a few minutes to transfer over a typical WLAN.

There are network analyzers and packet capture programs such as Novell's Lanalyzer that also provide throughput numbers by looking at all packets being transferred through the network. Of course, some application has to be using the network and saturating the capacity to get meaningful numbers. We often use this technique for analyzing network management packets and specific circumstances of network usage by programs. These can be used in conjunction with the FTP file transfer method described above. There are applications such as TTCP [8], iPerf [9] and Qcheck [10] that are specifically designed for network testing and are publicly available as shareware or freeware. We use all three and compare their results to the FTP benchmarks. These crosschecks are very valuable, because under certain circumstances, one measurement tool may produce very different results from the others. Knowing the detailed mechanisms used by these tools for benchmarking performance allows a skilled network engineer to infer the basic cause of such anomalous phenomenon.

The PCATTCP product is a port of the original BSD tool TTCP for use at a Windows command prompt to initiate winsock socket connections at the application layer between the client and server. In a crosscheck of validation between iPerf and PCATTCP, results showed very similar throughput numbers with PCATTCP slightly lower in all but one test configuration. iPerf was chosen as the preferred method of testing not as a result of these validation tests, but for providing greater ease of use and a wider availability of testing options.

Using iPerf in a client/server mode involved setting up a laptop computer on both ends of the link to be tested and running the program from the client side. Throughput numbers were recorded in most cases as an average of several iterations or as the highest and lowest observed values over a minimum of 3 iterations. Default mode of operation is to send 16776000 bytes of data from client to server and record the time it takes to send. Throughput is calculated by dividing the amount of data sent by the time taken to send it.

NetIQ Qcheck works in a similar manner except that it is driven by a graphical interface and only needs endpoint software to be installed rather than running the program on both ends. Qcheck sends a default of 100 bytes during testing and throughput calculations are made in the same manner as iPerf. From a white paper describing the tool:

Calculations of throughput and response time are based on the number of bytes sent and received from the application's perspective. Qcheck's throughput measurement is lower than the throughput that you would see with a network protocol analyzer. Qcheck measurements reflect only the data payload - what an application user would see - without the protocol overhead, such as headers, trailers, flow control, connection setup, and retransmissions. [11]

Qcheck generated a wider range of values in testing, presumably from the smaller amount of data being sent, so results from a minimum of 5 iterations were averaged during throughput data collection. The fundamental difference between Qcheck and iPerf, which may have led to differing results during our tests, is in the amount of data they send. This affects the duration of the test and therefore the averaging interval. iPperf transmits a set amount of information and determines the time it takes for the information to be received, while Qcheck sends an information stream for a set period of time at a predetermined window size and determines how much is received. 


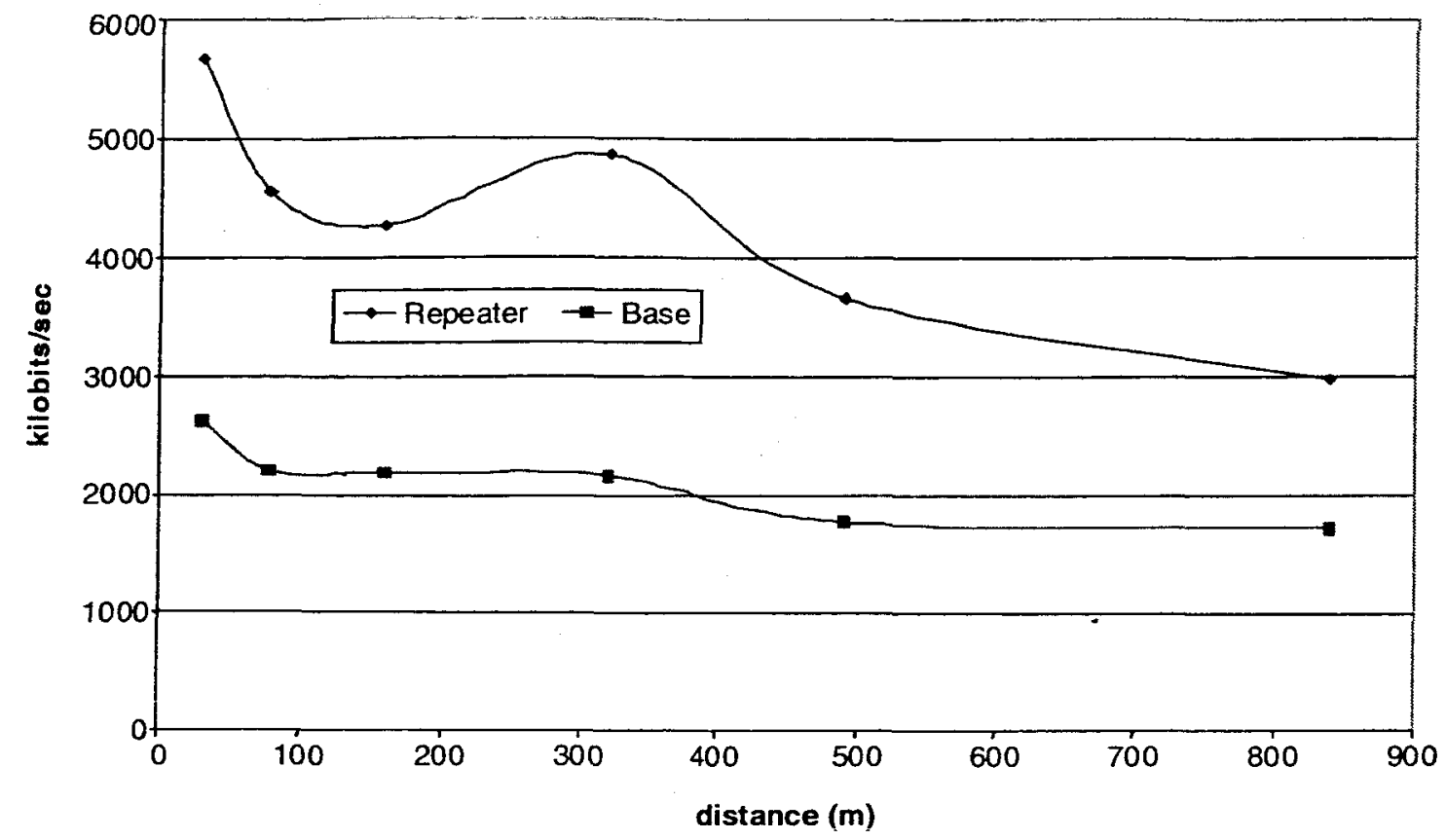

Graph 4 - Network Throughput - Sector Panel to Dipole

\section{Network Throughput Test Results}

The most important information that comes out of network throughput benchmarking is the ability to understand the sustainable throughput available to applications for a given WLAN configuration. The number of variables in WLAN performance makes it nearly impossible to predict performance under actual field conditions particularly for long links incorporating repeaters. However, the network throughput results obtained from our calibration tests do produce firstorder correlation of network throughput with RF signal levels, allowing prediction of network performance for a given link segment. Network throughput tests are performed at every data point right after the RF measurements are made, without changing anything. Network throughput is rather predictable and repeatable at short distances, but this is not true at longer distances. The MEX backbone is also a repeaterbased system, with up to two repeaters used to extend the range of the system. Repeaters can have a remarkable effect on network throughput, especially if they are half-duplex mode. The Cisco bridges operate as half-duplex repeaters, only being able to either transmit or receive at any given time. [12] They spend half the time receiving an incoming packet and the other half re-transmitting it to the far end of the chain. Half duplex essentially halves the throughput by reducing the duty factor by two.

There are two distinct mechanisms by which WLAN throughput is reduced. The first mechanism being a high BERR, resulting in packet loss, necessitating packet retransmission, and reducing throughput. The second is the effect of variable latency, primarily caused by processing delays in long WLAN chains of multiple repeaters. Latency effects are also caused by packet loss though, so the two effects can interact to reduce throughput even more. Increasing the signal strength or quality can mitigate the first effect by reducing BERR and incrcasing TCP/IP window size can reduce the second effect. A quantitative analysis of WLAN throughput under various conditions allows separating the two causes and optimizing both parameters properly.

The MEX project, using iPerf and Qcheck, measures network throughput and latency at various distances in our outdoor test range, varying the antenna configurations to generate a wide range of performance data for each WLAN product line. Two network throughput curves are generated, the first for the ATV to repeater link L2 (the same link tested for RF signal strength), and the second for the aggregate throughput through Link L1 and L2, from ATV through repeater to base.

The same test cases used for RF domain analysis are used to demonstrate the effect of link segment distance on network throughput. There are multiple effects that produce rather complex behavior for these graphs. The first case is the repeater-to-ATV link, using a sector panel-to-dipole antenna configuration shown in Graph 4, above, corresponding directly with the RF signal strength example. The top curve (repeater) shows a typical graph of diminishing throughput with increasing distance between ATV and repeater, repre- 
senting the Link 2 under test. A maximum of $5.9 \mathrm{Mbps}$ is obtained, nearly the maximum expected from an $11 \mathrm{Mbps}$ raw data rate WLAN link. WLAN protocols have a great deal of overhead and usually can only fill the raw data pipe by about $50 \%$. A typical figure for a wired LAN is about $80 \%$. The throughput hits a peak around 300 meters, another typical behavior observed in previous $802.11 \mathrm{~b}$ tests. [13] Finally, at longer distances, the graph shows a linear decline of throughput with distance, again fairly typical for WLANs.

The lower curve shows the throughput from the ATV through the repeater to the base. It exhibits half the throughput of the top one, graphically illustrating the factor of two reduction of throughput that occurs using a half-duplex repeater. However, at longer distances this relation no longer holds true, the throughput with the repeater is a bit better than a factor of two. This is because the longer link L2 dominates the throughput measurement due to its higher latency compared to the shorter repeater to base link.
The second test case (see Graph 5, below) involves the use of a Yagi antenna at the ATV, resulting in significant extra RF signal for L2 and greater throughput at farther points. For example, in the top curve for $\mathrm{L} 2,4000 \mathrm{Kbps}$ is achieved at 620 meters with the Yagi and at only 400 meters for the dipole, showing correlation of network throughput with RF signal strength. The bottom curve, ATV to base shows 2000 Kbps at $350 \mathrm{~m}$ for the dipole and $580 \mathrm{~m}$ for the Yagi. However, there are more complex phenomena at work. At 840 meters, the Yagi is showing somewhat decreased throughput compared to the dipole, with both $\mathrm{L} 2$ and $\mathrm{L} 1+\mathrm{L} 2$ data points showing lower numbers than expected.

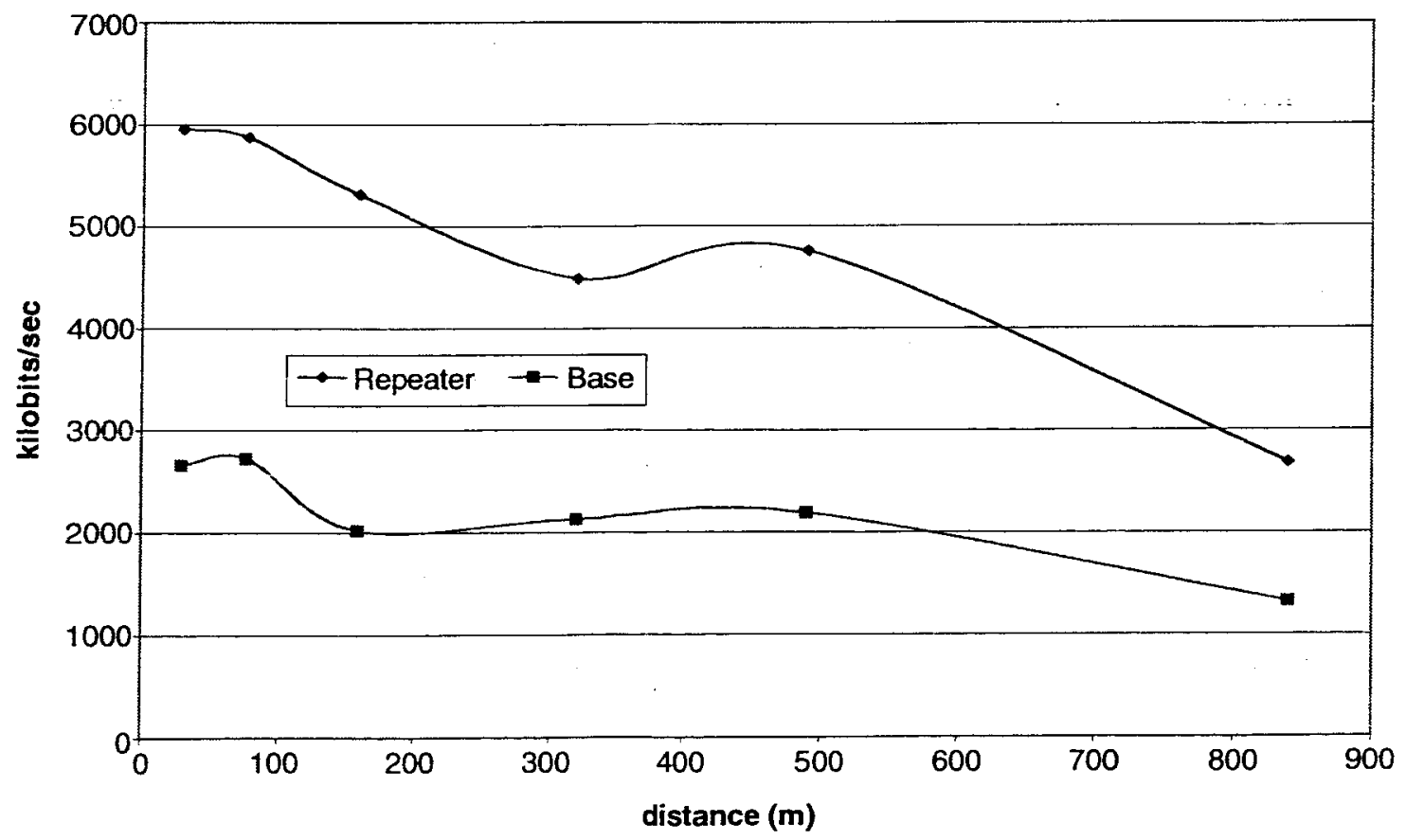

Graph 5 - Network Throughput -- Sector Panel to Yagi 


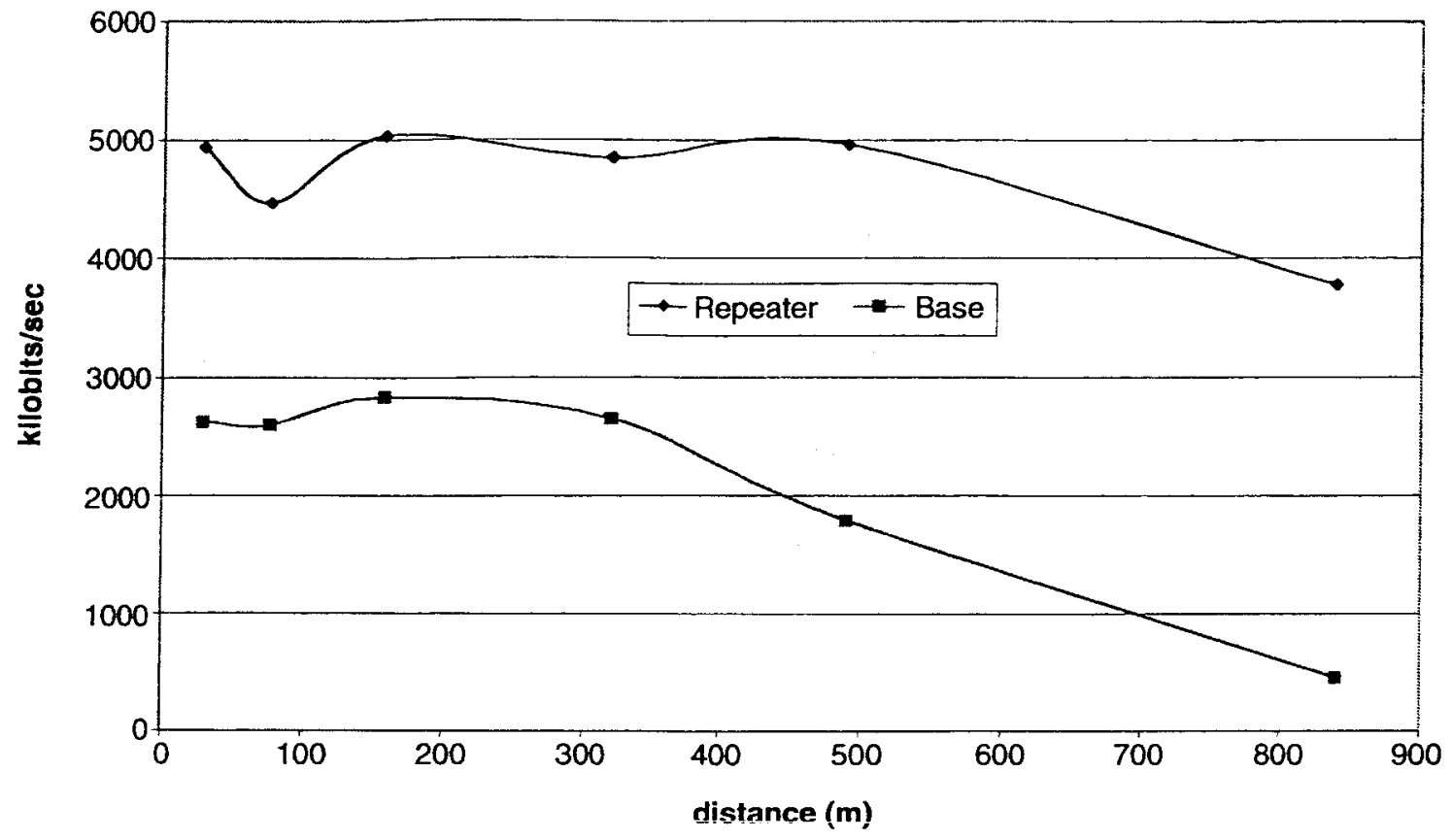

Graph 6 - Network Throughput - Sector Panel to Yagi, New Firmware

The third test case uses the same Yagi antenna as the second test case, but with upgraded bridge firmware. This is the same upgrade that solved the RF signal level measurement error, and many changes to the MAC layer timing were also made, necessitating generation of more throughput test results for the new firmware. Graph 6, above, shows the results of just the firmware upgrade on the network throughput, since all other equipment remained the same.

We observe a real increase in network throughput for $\mathrm{L} 2$ shown in the top curve. The numbers just stay higher over more distance. The same is true for the $\mathrm{L} 1+\mathrm{L} 2$ curve at short distances; however, at $500 \mathrm{~m}$ and at $840 \mathrm{~m}$, the throughput from ATV through repeater to base is significantly lower than it should be. The throughput at $500 \mathrm{~m}$ should be $2400 \mathrm{Kbps}$, but it is $1800 \mathrm{Kbps}$. At $840 \mathrm{~m}$, it should be $1800 \mathrm{Kbps}$, but is only $460 \mathrm{Kbps}$. This number is an average of the results of iPerf and Qcheck and is accurate. There is an effect occurring in the test range at the furthest data point which reduces throughput for certain tests, and it is not repeatable from test to test. We have reviewed all experimental controls and find that the variation of throughput has some patterns and is not the result of experimental error.

One hypothesis is that ground scatter reduces throughput, and further analysis was performed by comparing multiple test results from the old firmware and new firmware test cases. The analysis produced an unexpected result. The far end throughput at $840 \mathrm{~m}$ using the old firmware produced generally lower results than expected, with quite a bit of variation.
In contrast, the new firmware produced results in which the L2 far-end throughput was generally much better. The firmware upgrade reduced throughput variations at the far end of L2, generally producing consistently higher throughput over multiple tests.

The old firmware gave good results for the combined L1+L2 throughput, generally over $1 \mathrm{Mbps}$. However, the new firmware gave consistently lower numbers for throughput back to the base through $\mathrm{L} 1+\mathrm{L} 2$, generally under $0.5 \mathrm{Mbps}$. Each individual segment, $\mathrm{L} 1$ or L2, provided high throughput but the combination did not! A new hypothesis is emerging from this, which can only be inferred from multiple tests. Since a firmware upgrade will not affect basic radio performance, but does affect MAC layer timing, we must conclude that these throughput variations are basically caused by the complex timing interactions occurring in the MAC layer responsible for RF packet acknowledgement, media access and coordination, and repeater turnaround time. Low throughput using the repeater could be caused by higher latencies resulting from the firmware upgrade. The conclusion is that throughput across a repeater chain can be affected by numerous factors, and that we do not yet understand nor control all these factors. Future work will include detailed timing measurements of various aspects of network data transfer employing both packet protocol analyzers and network protocol analyzers for the MAC and TCP/IP layers respectively. 
Finally, we ran our full configuration consisting of two repeaters. For this test, we placed a second repeater at the farthest point along Link 2 at $840 \mathrm{~m}$ and varied the distance of a third link L3, perpendicular to link L2. Link L3 ran the length of another remote road with no obstacles. The first repeater now used two $18 \mathrm{dBi}$ small-dish grid antennas on a single mast, each aligned for best signal strength, while the second repeater used an $18 \mathrm{dBi}$ small-dish antenna along L2 and a $13 \mathrm{dBi}$ sector-panel antenna for link L3 to the Yagi mounted on the ATV. Waypoints were established and data taken at 100,200 and 300 meters for L3. Link L.2 was fixed at 840 meters of length.

Table 5, below, shows the results of two test runs at 300 meters for L3 using the old firmware. The RF signal strength was very good for both $\mathrm{L} 2$ and $\mathrm{L} 3$ and the corresponding throughput numbers are also quite high. Observe that the first repeater generally results in a reduction of a factor of two in throughput due to half duplex duty cycle, but that the reduction of adding the second repeater is not another factor of two, which might be expected. Rather, there is some overlap between the two repeaters' duty cycles which reduces the throughput reduction effect of the second repeater. The overlap is about $50 \%$, which significantly improves throughput for multiple repeater chains. For these tests, it is important that the test range be large enough for the ATV to be unaffected by the first repeater or the base station.

\section{Latency and Window Size}

Latency is defined as the response time for transferring packet data. This is a complex interaction of the transmit time, the round-trip-time (RTT) of the physical medium (light speed delay s) and the time required to send an acknowledgement. Light speed delays would be on the order of 3 usec for a 1 $\mathrm{Km}$ link, and computer response times are on the order of 1 msec or less. Of more significance are the data buffers in various network components that increase transport delay times as well as related processing steps such as generating an acknowledgement. We use a feature of Qcheck that sends $16 \mathrm{~KB}$ of data and measures the response time. For a single WLAN hop of $840 \mathrm{~m}$, we see latencies of about $34 \mathrm{msec}$ with about one of five measurements yielding higher numbers between 34 to $100 \mathrm{msec}$. The maximum seen was $150 \mathrm{msec}$. This latency scales directly with link length and is dominated by processing delays in the network hardware.

A basic primer in network TCP/IP function provides insight into a particular behavior of these network stacks that has a direct impact on network throughput. The "window size" is the size of the network buffer used to aggregate data into a series of TCP/IP packets that require a single acknowledgement. For example, MicroSoft Windows uses $8 \mathrm{~KB}$ window sizes by default. For $1.5 \mathrm{~KB}$ packet sizes typical of WLAN MAC layers, this translates to about 6-7 packets of data being sent before the recipient returns a single acknowledgement to the sender. If any errors occur in the transmission, the entire $8 \mathrm{~KB}$ buffer is sent again, incurring a significant throughput penalty because this doubles the time required to send that $8 \mathrm{~KB}$ chunk of the file, halving the throughput. Neither the MAC layer nor the TCP/IP layer returns errors in the event of single packet loss; rather each layer automatically resends the packet and does not report statistics on this process. Only if the loss of packet transfer is persistent do these layers report an error and abort the transfer. Therefore, highlevel application layer TCP/IP network bandwidth measurements are used to provide indirect measurements of MAC layer error rates and performance characteristics.

There is another consequence of window size with respect to network latency that causes significant network throughput problems, particularly with satellite links or long WLAN segments. Recall that one window-size buffer is sent and requires a single acknowledgement that is received after the RTT delay of the link. For an $8 \mathrm{~KB}$ window-size default buffer and 1 msec RTT delay typical of wired LANs, this produces a theoretical maximum throughput of $8 \mathrm{~KB} / \mathrm{msec}$ or $8 \mathrm{MB} / \mathrm{sec}$ or 64 Mbits/sec (Mbps). Obviously, this will not create a problem for medium performance wetworks with only $100 \mathrm{Mbp}$ : of raw data rate. However, note what happens on a space-based satellite link to a geosynchronous-orbit satellite. The distance is over 50,000 miles, incurring a light-speed delay of about 0.6 seconds round-trip. In this case, the $8 \mathrm{~KB}$ window size produces $8 \mathrm{~KB} / 0.6 \mathrm{sec}$ or $13 \mathrm{~KB} / \mathrm{sec}$ for only $107 \mathrm{Kbps}$, far below the communication link data rate for many satellite links. Therefore, the effect of small window size interacting with communication latency can be the most significant variable in satellite communications. The obvious solution is to increase window size, something done in the registry or network stack parameter file.

Table 5. Two-Repeater Configuration Throughput

\begin{tabular}{c|c|c|c} 
Measurement & $\mathrm{L1}+\mathrm{L} 2+\mathrm{L3}$ & $\mathrm{L} 2+\mathrm{L3}$ & $\mathrm{L3}$ \\
\hline Signal Strength $(\mathrm{dBm})$ & & $-33(\mathrm{~L} 2)$ & -45 \\
\hline Throughput1 (Kbps) & 1388 & 1897 & 3599 \\
\hline Throughput2 (Kbps) & 1610 & 2756 & 4664 \\
\hline
\end{tabular}


Table 6 - Network Throughputfor Various Latency and Window-size Values

\begin{tabular}{r|c|c|c|c} 
Latency (msec) & 8KB Window (Kbps) & 16KB Window (Kbps) & 32 KB Window (Kbps) & Typical for \\
\hline 1 & 64000 & 128000 & 256000 & LAN \\
\hline 10 & 6400 & 12800 & 25600 & WLAN short link \\
\hline 50 & 1280 & 2560 & 5120 & WLAN long link \\
\hline 100 & 640 & 1280 & 2560 & WLAN backbone \\
\hline 200 & 320 & 640 & 1280 & \\
\hline 400 & 160 & 320 & 640 & \\
\hline 600 & 107 & 213 & 427 & Geosync Satellite \\
\hline 800 & 80 & 160 & 320 & \\
\hline 1000 & 64 & 128 & 256 & \\
\end{tabular}

Window size cannot be increased arbitrarily however. If a single bit error occurs in the transmission of the buffer, the entire buffer must be retransmitted. Therefore, for any communication link there is an optimum setting, which balances network throughput restrictions caused by too small a window size with the throughput reduction caused by a high retransmission rate. The same analysis can be applied to WLANs, which have latencies ranging from tens of milliseconds to hundreds of milliseconds. We have seen latencies of $500 \mathrm{msec}$ on links employing multiple repeaters. Table 6, above, shows the bandwidth restrictions caused by latency effects using $8 \mathrm{~KB}, 16 \mathrm{~KB}$ and $32 \mathrm{~KB}$ buffers. This indicates that larger buffers are more appropriate for wireless links. Increasing window size to $16 \mathrm{~KB}$ in our WLAN tests has generally raised throughput $10 \%$, indicating average latencies are still rather short. Perhaps this effect explains the reduction in net throughput through a repeater that was observed with the new firmware. If the new firmware increases overall latency of data transfer, by slowing down repeater tumaround for example, this would appear as a reduction of net throughput even though the individual segments have high throughput. For a restriction to only $500 \mathrm{Kbps}$ for a default $8 \mathrm{~KB}$ window size, the average $\mathrm{L} 1+\mathrm{L} 2$ latency would have to be around $150 \mathrm{msec}$, which is not an unreasonable value. More testing will be performed to confirm this possible explanation.

\section{WLAN DESIGN AND DEPLOYMENT}

The design and field deployment of a WLAN system draws upon all of the domains presented. The RF analysis is applied at the design phase to ensure link strength and quality for the chosen link distance. This means calculation of link margin to ensure that adequate antennas are being specified together with antenna mounting masts of sufficient height to reduce the effects of the Fresnel zone for the range desired. One should always strive to reduce parasitic losses by using minimum cable lengths and high-quality cables and connectors. Adequate quality control of components is needed to prevent significant loss of radio signal strength due to sloppy construction of cables. Parasitic losses are significant and must be properly estimated for the link calculation. Output transmit power, receiver input sensitivity and antenna gain specifications are also needed to complete the link calculation. Large margins for RF link strength should be allowed, since theoretical calculations neglect many losses that occur in the field. The design phase is completed once the system is specified and expected performance detailed

During field deployment and testing, the design information can be used to determine how well a specific link is performing. This is done by comparing the field test results to design values or expected results. For example, our MEX $2003 \mathrm{sec}$ ond repeater hop covered a distance of over 2.6 miles between two high hills using two grid dish antennas. At this distance, one could not see the other repeater and even a spotting scope did not resolve the thin antenna mast on the far hill. We had trouble actually identifying which hill the repeater was mounted on at that distance, since there were so many hills at the Utah field site. Figure 4, next page, shows the far end repeater and antennas, with the sector panel antenna completing the link to the ATV. We used the antennaalignment tool from the second repeater location to determine RF signal strength numbers for this long link, and used it for pointing the antenna. The procedure was to first optically align the antennas by eye, then use the antenna alignment tool to adjust azimuth and elevation until maximum signal was obtained. However, both antennas needed to be pointed accurately to obtain best performance, so the far antenna was aligned by a second communication engineer at the far site communicating with the first engineer using a walkie talkie. This procedure is labor intensive and small adjustments to antenna alignment can have a big effect on RF signal strength at large distances. For example, we were able to go from $1 \%$ signal strength to $18 \%$ signal strength by simply aligning the antennas accurately. This was about a 10 -fold increase in actual signal power.

How well did we do on the repeater placement and antenna alignment? We measured the RF signal strength at the second repeater site at $-86 \mathrm{dBm}$ using the antenna alignment tool. This resulted in $18 \%$ signal strength and good signal 
quality numbers. If we extrapolate from the calibration curves we find that this measured value corresponds to roughly- 68 $\mathrm{dBm}$ theoretical signal strength at the far end of the link. Here's where we tum the equations around and determine the predicted theoretical range corresponding to this theoretical signal strength using two $18 \mathrm{dBi}$ grid antennas. This predicted distance was 4.9 miles compared to an actual distance of 2.6 miles. We achieved over $55 \%$ of predicted range, a reasonable value for rough terrain. In $\mathrm{dB}$ values, we lost about $6 \mathrm{~dB}$ of link margin (half the distance needs one-quarter the power) beyond theoretical, which means there were no gross errors in setup or alignment. This value also gives us an approximation for additional losses over this distance.

Our throughput calibration curves can give us some indication of expected throughput under various field conditions. We simply find the comparable RF field strength number and look at the corresponding throughput that resulted. This is an approximation, but a reasonable one if applied to a single link segment. The distances must also be comparabie for this to be true, for similar RTT. A very rough interpretation applied to the second repeater hop described above would yield a link segment throughput of between 2 to $3 \mathrm{Mbps}$. This would correspond to a network throughput from habitat to ATV of about 0.5 to $1 \mathrm{Mbps}$, based on the double repeater calibration tests. Our only problem was that after a strong wind came up

\section{Figure 4}

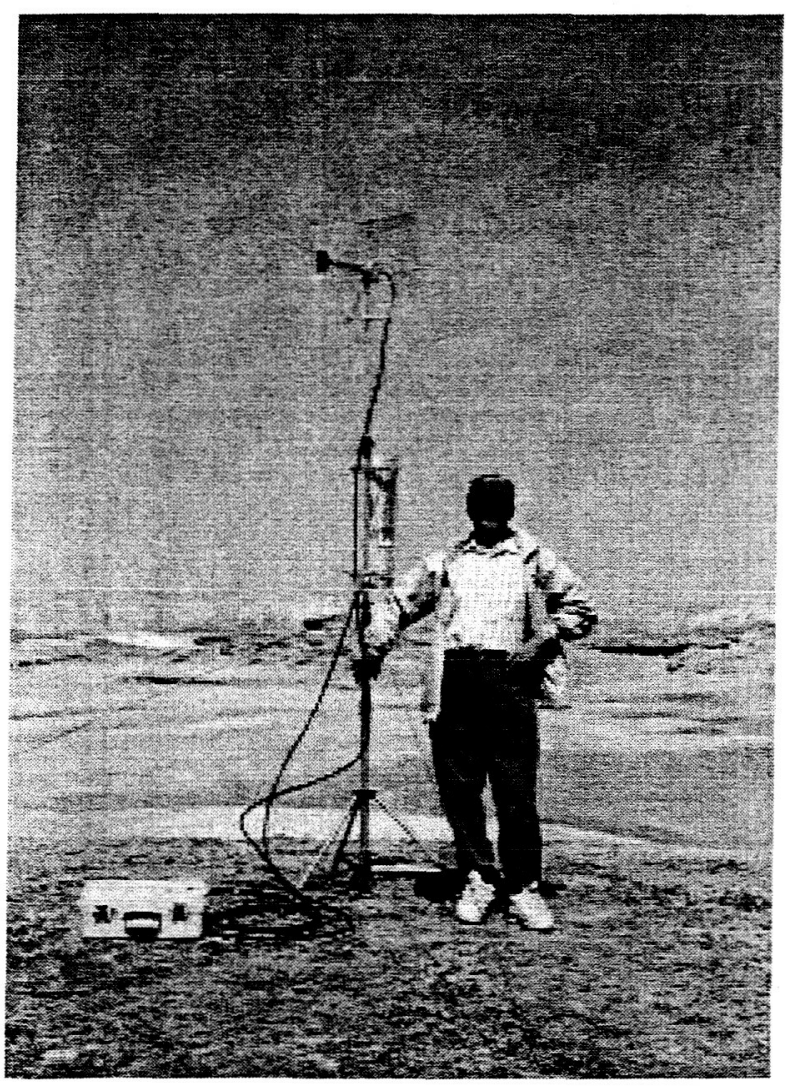

in the afternoon, the repeater masts and antennas started swaying and this was reflected in network throughput fluctuating in sync with the swaying!

While rough estimation of throughput can be achieved by extrapolating the calibration data, significant variability in end-to-end network throughput has been observed under a wide variety of conditions particularly at longer distances. The working hypothesis is that these variations are caused by long and highly variable latencies in packet transmission caused by timing interactions and/or high BERR primarily at the MAC layer, but also in the TCP/IP layer and applications. Most of the time, we see that and Qcheck provide very similar values for throughput, but occasionally they differ significantly. When this occurs, we take many throughput measurements using both tools and even repeat the entire calibration run to ensure accuracy. The throughput measurements presented in the graphs represent an average of several test measurements using both iPERF and Qcheck. The variations persist and are repeatable, from a statistical viewpoint. We need detailed and accurate measurements of latency at numerous points in the WLAN system to analyze this phenomenon.

The variability of WLAN throughput performance despite stringent controls placed on our test range supports our empirical method for WLAN metrics. There are too many unpredictable timing variations and interactions which couple with a variety of packet data loss mechanisms to allow linear analysis. This leaves detailed modeling and simulation and controlled field tests as the only methods that work for these highly complex systems. It is recommended that network throughput and latency measurements be employed to bench-mark every WLAN installation particularly for long-haul remote field use. The MEX project is looking at automating WLAN metrics data collection.

Figure 5

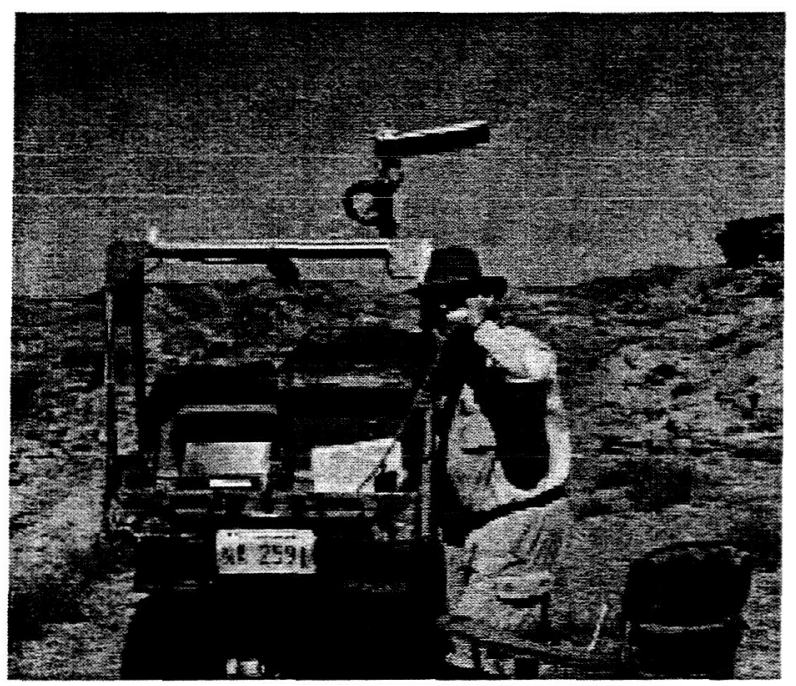


Figure 5 (preceding page) shows the author placing a phone call over the MEX backbone WLAN to the habitat over 5 miles away. At this distance, our walkie' talkies would not work and this was the only way to speak to people in the habitat to coordinate the simulations. We had good voice quality on the VoIP telephones concurrent with inter-agent messages being sent by the Mobile Agents software, with occasional background FTP file transfers. The network performed its required functions and even supported the voice link.

\section{CONCLUSION}

The MEX project has defined a pragmatic method of WLAN analysis and engineering applicable to a wide range of product lines and specifically addressing long-haul WLANs for use in rugged terrain. A method of experimentally reconciling WLAN site measurement tools with radio theory has been developed that correlates well with data from field deployment. Calibration curves that normalize WLAN RF signal strength data with theoretical calculations allow numerous site survey tools supplied by WLAN product vendors to be applied with consistency and certainty even in hybrid networks. Furthermore, the methods can be used to normalize and calibrate a variety of instruments including packet analyzers and spectrum analyzers. Additional losses due to Fresnel zone obstruction, atmospheric absorption and multipath distortion must be accounted for, requiring signifjcant additional margins in the RF link calculations.

The correlation of network throughput to RF signal strength is valid only for a single link segment provided the link distance and antenna configuration are the same. The throughput measurements are not repeatable at long distances or through multiple repeater chains due to factors that appear to be complex timing interactions at the MAC layer. These timing interactions are affected by link distances and vary even if the physical configuration of the WLAN remains constant. Despite maintaining fully controlled test conditions, we saw variations in throughput between measurements and from test run to test run that could not be explained. There was a statistical pattern to the variations, indicating this was not a measurement artifact or error. For example, the $840 \mathrm{~m}$ throughput was low for 5 of 10 measurements using the old firmware but for only 2 of 10 using the new firmware and this persisted over several runs performed on different days. Although RF signal strength was very consistent and repeatable from test to test, throughput was not. Consistent RF signal strength validated our experimental controls, so throughput variations were not an artifact of the test range or procedures.

In summary, WLAN design is best accomplished by using RF link analysis to assure adequate link margin. Subsequently, these RF signal strength margins can be verified in the field to validate the effectiveness of the field setup and alignment. Throughput in such complex hybrid WLAN systems is best determined experimentally to give software developers and application end-users some expectation of network performance. Throughput may vary even for fixed WLAN configurations and this needs to be taken into account by applications.

\section{REFERENCES}

[1] Stephen Braham, Peter Anderson, Pascal Lee, Richard Alena, and Brian Glass, "Canada and Analogue Sites for Mars Exploration," 1999 Proceedings of the Second Canadian Space Exploration Workshop, http://www.marsonearth.org.

[2] M. Sierhuis, M. H. Sims, W. J. Clancey, and Pascal Lee. "Applying multiagent simulation to planetary surface operations," in L. Chaudron (Ed.), COOP 2000 Workshop on Modelling Human Activity. Sophia Antipolis, France, pp. 19-28, 2000.

[3] Cisco Systems Web site. http://www.cisco.com

[4] W. Tomasi. Electronic Communications Systems: Fundamentals through Advanced. Prentice Hall, USA, 1998.

[5] Cisco Systems Web site. http://www.cisco.com

[6] R. Alena, D. Evenson, V. Rundquist, "Analysis and Testing of Mobile Wireless Networks" IEEE Aerospace Conference 2002.

[7] R. Alena, E. Yaprak, and S. Lamouri, "Modeling A Wireless Network For International Space Station," 1999 IEEE Aerospace Conference Proceedings, March, 1999.

[8] The Story of the TTCP Program Web site.h ttp://ftp.arl mil/ $\sim$ mike/ttcp.html

[9] NLANR Distributed Applications Support Team Web site. http://dast.nlanr.net/Projects/Iperf/

[10] Ixia Enterprise Qcheck Web site. http://www.netiq.com/ Qcheck

[11] Jeffrey T. Hicks and John Q. Walker, "A Quick Check of Network Performance," Web site.

[12] R. Alena, B. Gilbaugh, and B. Glass, "Communication System Architecture for Planetary Exploration," 2001 IEEE Aerospace Conference, March , 2001.

[13] R. Alena, D. Evenson, V. Rundquist, "Analysis and Testing of Mobile Wireless Networks," 2002 IEEE Aerospace Conference, March, 2002. 


\section{BIOGRAPHIES}

Richard Alena is a Computer Engineer and Group Lead for the Intelligent Mobile Technologies (IMT) Lab and the Mobile Exploration System (MEX) testbed at NASA Ames Research Center. The IMT team integrates mobile hardware and software components into unique systems capable of extending human performance aboard spacecraft during flight. He was principal investigator for the Wireless Network Experiment flown aboard Shuttle and Mir, technology later adopted by the International Space Station Program. He is a co-investigator on the Mobile Agents project, which is conducting field simulations in the American southwest. He has a MSEE\&CS from University of California, Berkeley.

Thom Stone is a Senior Computer Scientist with Computer Sciences Corporation. He is attached to the NREN (NASA Research and Education Network) project at Ames Research Center (ARC), where he has been employed by various contractors since 1989. He was an engineer with the NASA Science Internet project office when he led the project that brought reliable Internet connections to remote locations, including US bases in Antarctica, McMurdo Station and Amundson Scott South Pole Station. He was principal engineer for communications for the NASA Search for Extraterrestrial Intelligence project and a senior engineer for the Space Station Biological Research Project (SSBRP). Before his NASA work, Stone was employed in the computer and communications industry and taught telecommunications at the undergraduate level.

John Ossenfort is a current intern at the NASA Ames Research Center and is near completion of a UNIX certification from Foothill College. He has been acting system administrator for the IMT Lab and the MEX testbed and accompanied the Mobile Agents team on two field simulations, assisting in all aspects of wireless network design, deployment, troubleshooting and maintenance. John has a dual BA degree in Anthropology and East Asian Studies from Washington University in St. Louis.

Charles Lee is a computer scientist employed by Scientific Applications Intel Company (SAIC) at NASA Ames Research Center. He is currently working on the MEX project in the Intelligent Mobile Technologies (IMT) Lab responsible for software development for distributed sensor agents. He received the PhD Degree in computer science and systems engineering from Oakland University. His research interests are machine learning, pattern recognition, object component methodologies and wireless network performance.

Ed Walker is an aspiring network engineer and intern working in the Intelligent Mobile Technologies (IMT) Lab at NASA Ames Research Center. He is a member of the team developing and testing the capabilities of the Mobile Exploration System (MEX) testbed. The MEX System is a model for human planetary exploration that incorporates rugged computing, long-range wireless communication and mobility in support of planetary explorers. He is a student at Foothill College in Los Altos, California where he is currently working on an AS in Data Communication \& Network Management as well as Microsoft $M C S E$ and Cisco CCNA certifications. 